University of Nebraska - Lincoln

DigitalCommons@University of Nebraska - Lincoln

$11-15-1998$

\title{
The effect of a uniform electric field on homogeneous vapor-liquid nucleation in a dipolar fluid. I. Stockmayer fluid
}

\author{
K.J. Oh \\ University of Nebraska-Lincoln \\ G.T. Gao \\ University of Nebraska-Lincoln \\ Xiao Cheng Zeng \\ University of Nebraska-Lincoln, xzeng1@unl.edu
}

Follow this and additional works at: https://digitalcommons.unl.edu/chemzeng

Part of the Chemistry Commons

Oh, K.J.; Gao, G.T.; and Zeng, Xiao Cheng, "The effect of a uniform electric field on homogeneous vapor-liquid nucleation in a dipolar fluid. I. Stockmayer fluid" (1998). Xiao Cheng Zeng Publications. 64. https://digitalcommons.unl.edu/chemzeng/64

This Article is brought to you for free and open access by the Published Research - Department of Chemistry at DigitalCommons@University of Nebraska - Lincoln. It has been accepted for inclusion in Xiao Cheng Zeng Publications by an authorized administrator of DigitalCommons@University of Nebraska - Lincoln. 


\title{
The effect of a uniform electric field on homogeneous vapor-liquid nucleation in a dipolar fluid. I. Stockmayer fluid
}

\author{
K. J. Oh, G. T. Gao, and X. C. Zeng ${ }^{\text {a) }}$ \\ Department of Chemistry, University of Nebraska-Lincoln, Lincoln, Nebraska 68588
}

(Received 7 July 1998; accepted 10 August 1998)

\begin{abstract}
The rate of homogeneous vapor-liquid nucleation is controlled mainly by the Gibbs formation free energy of critical clusters. Within the framework of the $i / v$ cluster theory of Reiss and co-workers, the formation free energies of Stockmayer clusters in the presence of a uniform electric field are determined. The Helmholtz free energy of the clusters, an input required in the $i / v$ cluster theory, is calculated via Monte Carlo simulations. The most significant result is that as a uniform electric field is applied the formation free energy at fixed supersaturation of the vapor becomes larger than that in the absence of a field, whereas at fixed chemical potential the formation free energy becomes smaller. (C) 1998 American Institute of Physics. [S0021-9606(98)50543-0]
\end{abstract}

\section{INTRODUCTION}

Electric fields play an important role in atmospheric processes. For example, it is well known that there exists a vertical electric field in a free and undisturbed atmosphere; intense electric fields can be generated in thunderclouds. ${ }^{1}$ From fundamental scientific point of view, the effect of a uniform electric field on vapor-liquid nucleation is also of interest, particularly in connection with the nucleation experiments. For example, by adjusting the strength of the electric field, one can vary the effective supersaturation of the system with high precision.

There have been several studies of the effects of an electric field on vapor-liquid nucleation. ${ }^{2-7}$ Many of those studies are concerned with ion-induced nucleation which also plays a major role in atmospheric condensation, especially in the ionosphere. Since the electric field strength of an ion decreases with increasing the distance from the ion, the electric field of an ion is nonuniform. Because of this nonuniformity, polar molecules of a supersaturated vapor tend to move toward the region of higher electric field, that is, the region near the ion. Thus, one can expect that vapor-liquid nucleation could be induced by the presence of the ion. The enhancement of the nucleation rate by ions has been indeed observed experimentally. ${ }^{2,3}$

The aim of this study is to explore the effects of a uniform electric field on the rate of vapor-liquid nucleation. The effects can be quite different from those due to ions because in the uniform field there is no preferential region for the dipolar molecules to move into, although the dipoles of the molecules tend to orient along the direction of the field. Kashchiev ${ }^{4}$ was the first to point out the importance, for studying the field effects, of considering the dielectric constants of both the cluster and supersaturated vapor in the presence of a uniform electric field. He argued that when the dielectric constant of the new phase is smaller than that of the old phase, the electric field stimulates the nucleation process, whereas in the opposite case it inhibits the process.

${ }^{a)}$ Electronic mail: xzeng@unlinfo.unl.edu
Contrary to Kashchiev's results, Isard ${ }^{5}$ claimed that the formation free energy of a cluster is always reduced in a uniform field, even when the dielectric constant of the cluster is greater than that of the supersaturated vapor. $\mathrm{Cheng}^{6}$ also showed that the electric field leads to a reduction of the size of the critical cluster as well as of the formation free energy. Similarly, it has been reported that the nucleation rate increases sharply under a high external magnetic field, ${ }^{8}$ and that the process of phase separation in a magnetic fluid goes on more rapidly in the presence of a higher magnetic field.

To our knowledge, most previous theoretical studies on electric field-induced nucleation invoked the capillarity approximation and, thus, the nucleus was treated as a dielectric continuum. Molecular details were not considered. Clearly, when the critical nucleus contains only a few tens of molecules, the applicability of the capillarity approximation is questionable. One such example is the orientational correlations that is shown to be important in vapor-liquid nucleation in polar fluids. ${ }^{9,10}$

In order to understand the effects of a uniform electric field on vapor-liquid nucleation, here we adopt a molecular approach-the $i / v$ cluster theory-developed by Reiss and co-workers. ${ }^{11-15}$ The essence of $i / v$ cluster theory is that the cluster (i.e., nucleus) is defined as a group of $i+1$ molecules (including the so-called shell molecule) within a container having volume $v$. The center of the spherical container coincides with the center of mass of $i+1$ molecules and the boundary of the container is marked by the location of the shell molecule that resides in the volume shell of the volume. The main difference between the $i / v$ cluster and an earlier Lee-Barker-Abraham (LBA) cluster is the introduction of the shell molecule. The latter removes much of the redundant counting of the cluster configurations inherent in the LBA cluster. ${ }^{16}$ Once the $i / v$ cluster is defined, the metastable equilibrium distribution of $i / v$ clusters can be obtained by minimizing the partition function with respect to the distribution. The $i / v$ cluster theory requires the input of the Helmholtz free energy of the cluster, which is readily obtained from a Monte Carlo simulation. ${ }^{13,15}$ 
In this work, we extend the $i / v$ cluster theory to a dipolar system in a uniform electric field. The dipolar interactions between molecules are modeled by the Stockmayer potential, which consists of a Lennard-Jones potential plus a dipoledipole potential. Monte Carlo simulation is used to calculate the Helmholtz free energy of clusters under the approximation of decoupling ${ }^{15}$ the shell molecule. The latter approximation renders the Monte Carlo (MC) simulation easier to implement.

\section{FORMATION FREE ENERGY OF CLUSTER}

In most vapor nucleation measurements the temperature is well below the critical point. Therefore the involved supersaturated vapor can be well approximated as an ideal gas. In other words, the intermolecular interaction between the cluster and the vapor can be ignored. As for the electrostatic contribution, if the surrounding vapor is considered as a continuum, interactions between the cluster and the surrounding vapor can be expressed in terms of the reaction field, which is given by ${ }^{17}$

$$
\mathbf{E}_{R}=\frac{1}{a^{3}} \frac{2 \epsilon-2}{2 \epsilon+1} \mathbf{M}
$$

where $a$ is the radius of the cluster, $\epsilon$ is the dielectric constant of the surrounding vapor, and $\mathbf{M}$ is the total dipole moment vector of the system. As such, the reaction field is very small if $\epsilon$ is close to 1 (dielectric constant of vacuum). In addition, a nucleation event is rare, that is, the large density fluctuations that lead to condensation are few enough and far enough apart so that they may be regarded as mutually decoupled, and the interference effects between clusters may be ignored. Thus, the partition function for entire supersaturated vapor may be written in the form

$$
Q=\sum_{\left\{\tilde{n}_{i+1}, v\right\}} \frac{\left[\gamma_{T} \gamma_{R} V_{1} q_{E}\right]^{N_{1}}}{N_{1} !} \prod_{i=1} \prod_{v} \frac{q_{i+1, v}^{\tilde{n}_{i+1, v}}}{\tilde{n}_{i+1, v} !},
$$

where $T$ is temperature and $\gamma_{T}=\left(2 \pi m k_{\mathrm{B}} T\right)^{3 / 2} / h^{3}, \quad \gamma_{R}$ $=\left(2 \pi I k_{\mathrm{B}} T\right) / h^{2}, m$ and $I$ are the mass and the moment of inertia of a molecule, respectively; $N_{1}$ is the number of molecules (monomer) not in the clusters, which is given by

$$
N_{1}=N-\sum_{i=1} \sum_{v}(i+1) \tilde{n}_{i+1, v},
$$

where $N$ is the total number of molecules in the system; $V_{1}$ is the volume available to the molecules of the vapor and is given by

$$
V_{1}=V-\sum_{i=1} \sum_{v} v \tilde{n}_{i+1, v}
$$

where $V$ is the volume of the system. In Eq. (2), $\left\{\tilde{n}_{i+1, v}\right\}$ refers to the set of numbers denoting a particular distribution of cluster sizes, there being $\tilde{n}_{i+1, v}$ clusters in that distribution. As indicated in the previous section, the cluster is defined by $i$ molecules within the volume $v$ whose surface is marked by a shell molecule, while its center coincides with the center of mass of the $i+1$ molecules and its partition function $q_{i+1, v}$ is given by

$$
\begin{aligned}
q_{i+1, v}= & \frac{(i+1)^{3} V \gamma_{T}^{i+1} \gamma_{R}^{i+1}}{i !} \\
& \times \int_{v} \cdots \int_{v} \int_{d v} e^{-U_{i+1} / k_{\mathrm{B}} T} d \mathbf{r}_{1}^{\prime} \cdots d \mathbf{r}_{i}^{\prime} d \mathbf{e}_{1} \cdots d \mathbf{e}_{i+1},
\end{aligned}
$$

where the primed coordinates are in the center of mass of the $i+1$ particles ( $i$ interior plus the shell molecules) and $U_{i+1}$ is the potential energy of the $i+1$ molecules that is given by

$$
U_{i+1}=U_{i+1}^{0}-\mathbf{M} \cdot \mathbf{E},
$$

where $U_{i+1}^{0}$ is the interaction energy in the absence of the electric field, $\mathbf{E}$ is the electric field, and $\mathbf{M}$ is the dipole moment of the cluster,

$$
\mathbf{M}=\mu \sum_{k} \mathbf{e}_{k}
$$

where $\mathbf{e}_{k}$ is the unit vector along the dipole moment of the $k$ th molecule.

Let $q_{E}$ represent the contribution to the partition function of the supersaturated vapor due to the presence of the electric field. For a linear molecule, $q_{E}$ is given by

$$
q_{E}=\int_{0}^{2 \pi} \int_{0}^{\pi} e^{\mu E \cos \theta / k_{\mathrm{B}} T} \sin \theta d \theta d \phi=\frac{2 \pi}{x}\left(e^{x}-e^{-x}\right),
$$

where $\theta$ is the angle between the dipole moment and the direction of the electric field, and $x=\mu E / k T$. In the absence of the electric field, $q_{E}=4 \pi$.

The chemical potential of the cluster can be obtained from the definition

$$
\mu_{i+1, v}=\left(\frac{\partial A}{\partial \tilde{n}_{i+1, v}}\right)_{V, T, E, \tilde{n}_{j, v^{\prime}}}, \quad\left(j \neq i+1, v^{\prime} \neq v\right),
$$

where $A$ is the Helmholtz free energy of the total supersaturated system, and is given by

$$
\begin{aligned}
A=-k_{\mathrm{B}} T \ln Q= & k_{\mathrm{B}} T N_{1}\left(\ln \frac{N_{1}}{\gamma_{T} \gamma_{R} V_{1} q_{E}}-1\right) \\
& +k_{\mathrm{B}} T \sum_{i=1} \sum_{v} \tilde{n}_{i+1, v}\left(\ln \frac{\tilde{n}_{i+1, v}}{q_{i+1, v}}-1\right) .
\end{aligned}
$$

Thus, the partial derivative of $A$ with respect to $\tilde{n}_{i+1, v}$ yields

$$
\mu_{i+1, v}=\frac{N_{1} k_{\mathrm{B}} T v}{V_{1}}+k_{\mathrm{B}} T \ln \frac{\tilde{n}_{i+1, v}}{q_{i+1, v}} \quad(i \geqslant 1)
$$

and

$$
\mu_{1}=k_{\mathrm{B}} T \ln \frac{N_{1}}{\gamma_{T} \gamma_{R} V_{1} q_{E}} .
$$

Here we note that $\mu_{i+1, v}$ and $\mu_{1}$ are, respectively, the chemical potential measured at $p_{i+1, v}$, the partial pressure of the 
$(i+1) / v$ cluster, and $p_{1}$, the partial pressure of the monomer. The partial pressures are calculated from the partial derivative of $A$ with respect to $V$ as follows:

$$
\begin{aligned}
p & =\left(\frac{\partial A}{\partial V}\right)_{N, T, E, \tilde{n}_{i+1, v}} \\
& =N_{1} k_{\mathrm{B}} T / V_{1}+\sum_{i=1} \sum_{v} \tilde{n}_{i+1, v} k_{\mathrm{B}} T / V=p_{1}+\sum_{i=1} \sum_{v} p_{i+1, v} .
\end{aligned}
$$

Note that $\Delta G_{i+1, v}$ can be derived by using the defining relation

$$
\Delta G_{i+1, v}=\mu_{i+1, v}\left(p_{1}\right)-(i+1) \mu_{1} .
$$

Here $\mu_{i+1, v}\left(p_{1}\right)$ is the chemical potential of the $(i+1) / v$ cluster at $p_{1}$,

$$
\begin{aligned}
\mu_{i+1, v}\left(p_{1}\right) & =\mu_{i+1, v}+k_{\mathrm{B}} T \ln \frac{p_{1}}{p_{i+1, v}} \\
& =\frac{N_{1} k_{\mathrm{B}} T v}{V_{1}}+k_{\mathrm{B}} T \ln \frac{\tilde{n}_{i+1, v}}{q_{i+1, v}}+k_{\mathrm{B}} T \ln \frac{p_{1}}{p_{i+1, v}} \\
& =\frac{N_{1} k_{\mathrm{B}} T v}{V_{1}}+k_{\mathrm{B}} T \ln \frac{N_{1} V}{q_{i+1, v} V_{1}},
\end{aligned}
$$

where we have used the relations $p_{1}=N_{1} k_{\mathrm{B}} T / V_{1}, p_{i+1, v}$ $=\tilde{n}_{i+1, v} k_{\mathrm{B}} T / V$. Therefore

$$
\Delta G_{i+1, v}=\frac{N_{1} k_{\mathrm{B}} T v}{V_{1}}+k_{\mathrm{B}} T \ln \frac{N_{1} V}{q_{i+1, v} V_{1}}-(i+1) \mu_{1} .
$$

\section{DECOUPLING OF SHELL MOLECULE}

We obtained in the previous section that

$$
\Delta G_{i+1, v}=A_{i+1, v}+\frac{N_{1} k_{\mathrm{B}} T v}{V_{1}}+k_{\mathrm{B}} T \ln \frac{N_{1} V}{V_{1}}-(i+1) \mu_{1},
$$

where we replace $-k_{\mathrm{B}} T \ln q_{i+1, v}$ by $A_{i+1, v}$. To calculate $A_{i+1, v}$ from Monte Carlo simulation we adopted the socalled decoupling approximation ${ }^{15}$ of the shell molecule from the $i / v$ cluster. To be specific, we first write $A_{i+1, v}$ as

$$
A_{i+1, v}=-k_{\mathrm{B}} T \ln \gamma_{T}(i+1)^{3 / 2} V+A_{i+1, v}^{*},
$$

where

$$
\begin{aligned}
A_{i+1, v}^{*}= & -k_{\mathrm{B}} T \ln \frac{(i+1)^{3 / 2} V \gamma_{T}^{i} \gamma_{R}^{i+1}}{i !} \\
& \times \int_{v} \cdots \int_{v} \int_{d v} e^{-U_{i+1} / k_{\mathrm{B}} T} d \mathbf{r}_{1}^{\prime} \cdots d \mathbf{r}_{i}^{\prime} d \mathbf{e}_{1} \cdots d \mathbf{e}_{i+1} .
\end{aligned}
$$

When the decoupling approximation is taken into account, that is, when the interaction between the shell molecule and the remaining $i$ molecules is neglected (i.e., $\quad U_{i+1}\left(\mathbf{r}_{1}^{\prime} \cdots \mathbf{r}_{i-1}^{\prime}, \mathbf{r}_{i}^{\prime}, \mathbf{e}_{1} \cdots \mathbf{e}_{i+1}\right) \approx U_{i}\left(\mathbf{r}_{1}^{\prime} \cdots \mathbf{r}_{i-1}^{\prime}, \mathbf{e}_{1} \cdots \mathbf{e}_{i}\right)$ $\left.-\mu \mathbf{e}_{i+1} \cdot \mathbf{E}\right), \quad A_{i+1, v}^{*}$ can be rewritten as

$$
\begin{aligned}
A_{i+1, v}^{*} \approx & -k_{\mathrm{B}} T \ln \frac{(i+1)^{3 / 2} V \gamma_{T}^{i} \gamma_{R}^{i+1}}{i !} \\
& \times \int_{v} \cdots \int_{v} \int_{d v} e^{-U_{i+1} / k_{\mathrm{B}} T} d \mathbf{r}_{1}^{\prime} \cdots d \mathbf{r}_{i}^{\prime} d \mathbf{e}_{1} \cdots d \mathbf{e}_{i+1} \\
= & -k_{\mathrm{B}} T \ln \frac{(i+1)^{3 / 2}}{i^{3 / 2}} \gamma_{T} \gamma_{R} q_{E} d v+A_{i, v}^{*},
\end{aligned}
$$

where

$$
\begin{aligned}
A_{i, v}^{*}= & -k_{\mathrm{B}} T \ln \frac{i^{3 / 2} V \gamma_{T}^{i-1} \gamma_{R}^{i}}{i !} \\
& \times \int_{v} \cdots \int_{v} e^{-U_{i} / k_{\mathrm{B}} T} d \mathbf{r}_{1}^{\prime} \cdots d \mathbf{r}_{i-1}^{\prime} d \mathbf{e}_{1} \cdots d \mathbf{e}_{i} .
\end{aligned}
$$

$A_{i, v}^{*}$ is the Helmholtz free energy of an $i / v$ cluster, which can be evaluated via computer simulation as that of a LBA cluster without a shell molecule. By combining Eqs. (17), (18), and (20), we obtain

$$
\begin{aligned}
\Delta G_{i+1, v}= & -k_{\mathrm{B}} T \ln \frac{(i+1)^{3 / 2} \gamma_{T} \gamma_{R} q_{E} d v}{i^{3 / 2}}+A_{i, v}^{*} \\
& -k_{\mathrm{B}} T \ln \gamma_{T}(i+1)^{3 / 2} V+\frac{N_{1} k_{\mathrm{B}} T v}{V_{1}} \\
& +k_{\mathrm{B}} T \ln \frac{N_{1} V}{V_{1}}-(i+1) \mu_{1} .
\end{aligned}
$$

The last equation is rearranged to yield

$$
\begin{aligned}
\Delta G_{i+1, v}= & A_{i, v}^{*}-k_{\mathrm{B}} T \ln \gamma_{T}(i+1)^{3 / 2} V+\frac{N_{1} k_{\mathrm{B}} T v}{V_{1}} \\
& +k_{\mathrm{B}} T \ln \frac{N_{1} V}{V_{1}}-i \mu_{1}-k_{\mathrm{B}} T \ln \frac{(i+1)^{3 / 2}}{i^{3 / 2}},
\end{aligned}
$$

where we assumed $N_{1} d v / V_{1} \sim 1$.

\section{MONTE CARLO SIMULATION}

The dipolar system is modeled as a Stockmayer fluid with potential function

$$
\begin{aligned}
u_{i j}= & 4 \epsilon\left[\left(\sigma / r_{i j}\right)^{12}-\left(\sigma / r_{i j}\right)^{6}\right]+\frac{\mu^{2}}{r_{i j}^{3}}\left[\mathbf{e}_{i} \cdot \mathbf{e}_{j}-3\left(\mathbf{e}_{i} \cdot \mathbf{r}_{i j}\right)\right. \\
& \left.\times\left(\mathbf{e}_{i} \cdot \mathbf{r}_{i j}\right) / r_{i j}^{2}\right]
\end{aligned}
$$

where $\mathbf{r}_{i j}=\mathbf{r}_{i}-\mathbf{r}_{j}$ and $r_{i j}=\left|\mathbf{r}_{i j}\right|$. The first and the second term on the right-hand side of the above equation represent the usual Lennard-Jones function and dipole-dipole interaction, respectively. Here, we chose $\epsilon / k=119.8 \mathrm{~K}$ and $\sigma=3.405 \AA$. As for the interaction of a dipole with the electric field, we used $-\mu E \cos \theta$, where $\theta$ is the angle between the dipole and the electric field. All molecules are confined in a spherical container with a hard wall. We use the same simulation procedure as Lee, Barker, and Abraham ${ }^{16}$ to generate configurations for the position and orientation of the molecules. Besides the usual Metropolis procedure, an additional acceptance criterion is enforced: if an attempted move 


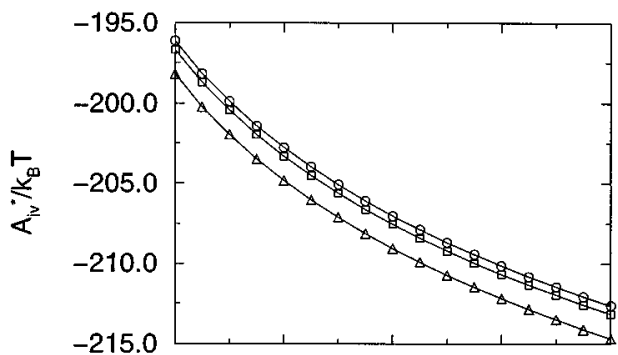

(a)

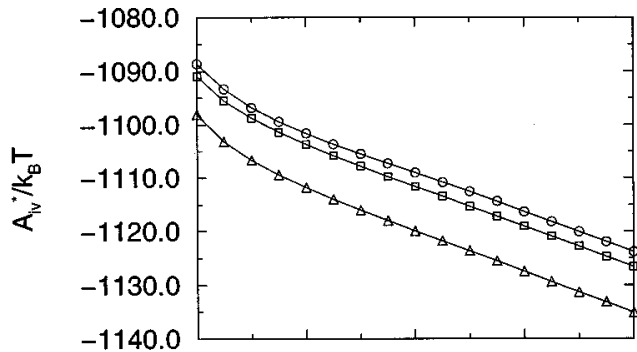

(d)

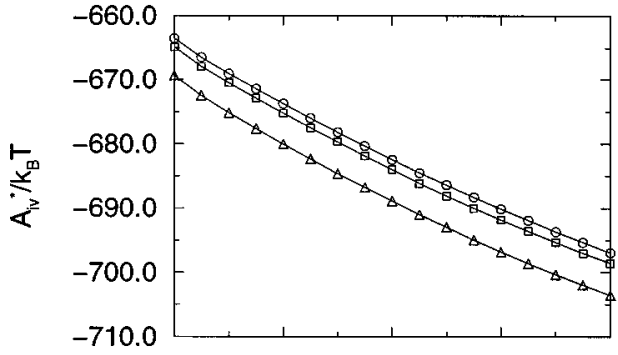

(b)

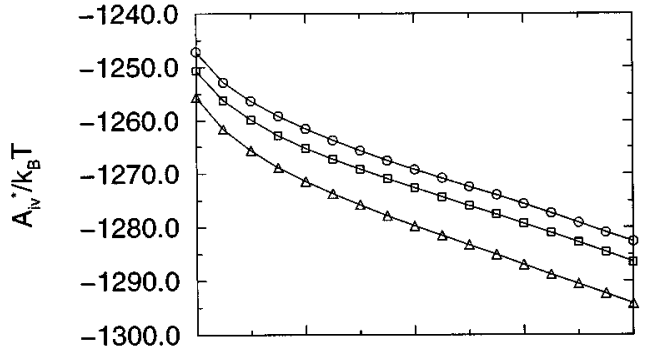

(e)
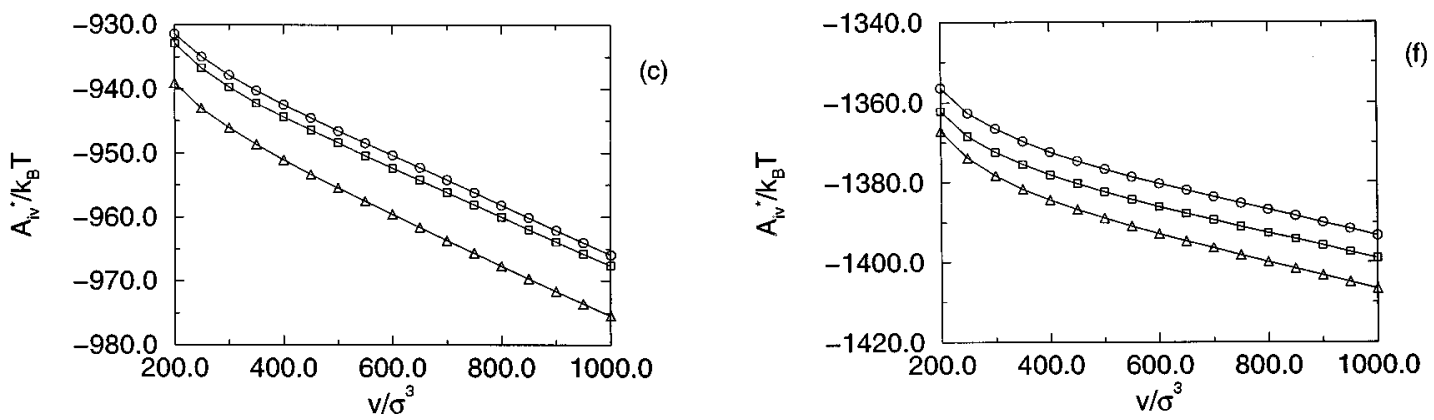

FIG. 1. $\Delta A_{i, v}^{*} / k_{\mathrm{B}} T$ as a function of container volume $v$ : (a) $i=13$, (b) $i=43$, (c) $i=60$, (d) $i=70$, (e) $i=80$, and (f) $i=87$ under $E=0.0\left(\sigma^{3} / \epsilon\right)^{1 / 2}$ (circle), $0.4\left(\sigma^{3} / \epsilon\right)^{1 / 2}$ (square), and $0.8\left(\sigma^{3} / \epsilon\right)^{1 / 2}$ (triangle up).

of the position of a molecule lead to any molecule being at a distance (from the new center of mass of $i$ molecules) greater than the radius of the container, this move would be rejected. No additional constraint is imposed on the orientational moves. The system is equilibrated after 50000 Monte Carlo steps per molecule. Data is collected through another 50000 Monte Carlo steps per molecule.

To compute $A_{i, v}^{*}$ in Monte Carlo simulations, we used the thermodynamic integration relation

$$
A_{i, v}^{*}=A_{i d}-\int_{v_{i d}}^{v} P d v,
$$

where $\quad P=(i-1) k_{\mathrm{B}} T / v+\langle W\rangle / v \quad$ and $\quad\langle W\rangle=-1 / 3 \Sigma_{i}$ $\sum_{j>i} r_{i j}\left[d u\left(r_{i j}\right) / d r_{i j}\right]$. The ideal gas is chosen as the reference state, and the Helmholtz free energy $A_{i d}$ is then given by

$$
\begin{aligned}
A_{i d}= & -k_{\mathrm{B}} T \ln \frac{4 \pi^{i} i^{3 / 2} \gamma_{T}^{i-1} \gamma_{R}^{i}}{i !} \\
& \times \int_{v_{i d}} e^{\mathbf{M} \cdot \mathbf{E} / k T} d \mathbf{r}_{1}^{\prime} \cdots d \mathbf{r}_{i-1}^{\prime} d \mathbf{e}_{1}^{\prime} \cdots d \mathbf{e}_{i}^{\prime},
\end{aligned}
$$

where $v_{i d}$ is the volume of the reference state, and where the integral $\int_{v_{i d}} d \mathbf{r}_{1}^{\prime} \cdots d \mathbf{r}_{i-1}^{\prime}$ gives $a(i) i^{-3 / 2} v_{i d}^{i-1}$, as shown in the Appendix of Ref. 16. The values of $a(i)$, as a function of $i$, range from 2.437 to a limiting value of 2.974 . Thus, $A_{i d}$ becomes

$$
A_{i d}=-k_{\mathrm{B}} T \ln \frac{a(i) \gamma_{T}^{i-1} v^{i-1} \gamma_{R}^{i} q_{E}^{i}}{i !} .
$$

To generate an ideal gas reference state, we chose $v_{i d}$ $=1000 i \sigma^{3}$ as the starting point in the thermodynamic integration. We found that at this $v_{i d} A_{i, v}^{*} / k_{\mathrm{B}} T$ is indeed close to the ideal gas value.

In all simulations, the molecular dipole moment $\mu$ is fixed at $1.414\left(1 / \epsilon \sigma^{3}\right)^{1 / 2}$ and temperature $T$ is fixed at $1.15 \epsilon / k_{\mathrm{B}}$. The molecular mass $m$ and moment of inertia $I$ are set to be $39.438 \mathrm{~g} / \mathrm{mol}$ and $19.3 \times 10^{-40} \mathrm{~g} \mathrm{~cm}^{2}$ for calculating $\gamma_{T}$ and $\gamma_{R}$.

\section{RESULTS AND DISCUSSION}

Figures $1(\mathrm{a})-1(\mathrm{~g})$ show $A_{i, v}^{*} / k_{\mathrm{B}} T$ curves for $i=13,43$, $60,70,80$, and 87 clusters with volumes ranging from $v$ $=200 \sigma^{3}$ to $v=1000 \sigma^{3}$ and under three different strengths of the electric field. As expected, $A_{i, v}^{*} / k_{\mathrm{B}} T$ decreases with the increasing electric field. 
(a)

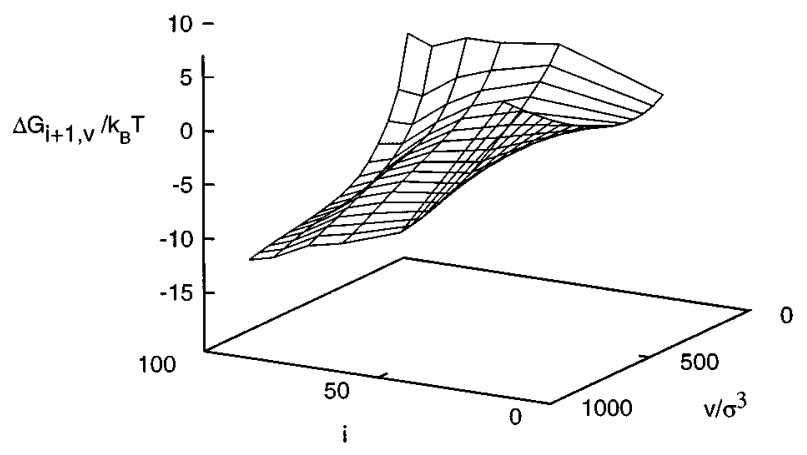

(b)

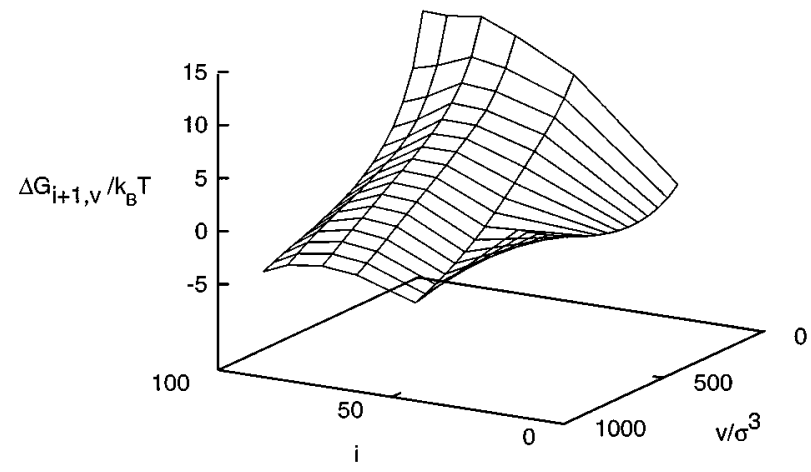

(c)

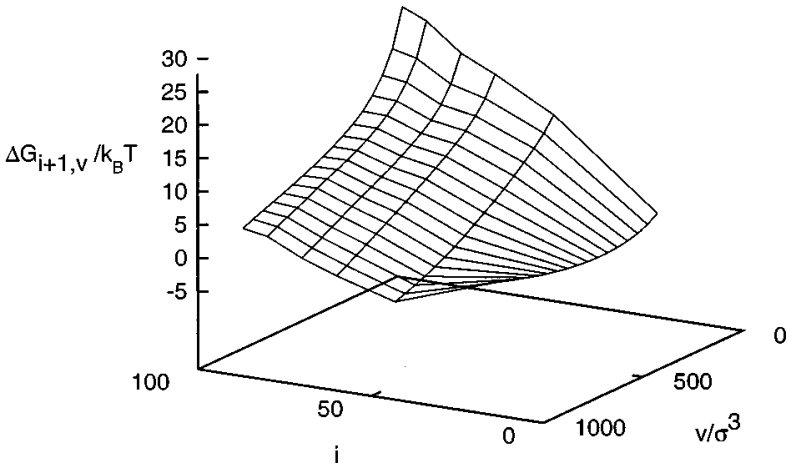

FIG. 2. $\Delta G_{i+1, v}$ surfaces for (a) $E=0.0\left(\sigma^{3} / \epsilon\right)^{1 / 2}$, (b) $E=0.4\left(\sigma^{3} / \epsilon\right)^{1 / 2}$, and (c) $E=0.8\left(\sigma^{3} / \epsilon\right)^{1 / 2}$.

Once $A_{i, v}^{*}$ is obtained from the Monte Carlo simulation, we can calculate $\Delta G_{i+1, v} / k_{\mathrm{B}} T$ using Eq. (23). Here we consider two different cases: fixed $p_{1} / p_{\text {eq }}$ (supersaturation) and fixed $\mu_{1}$ (monomer chemical potential). $p_{\text {eq }}$ is the coexistence vapor pressure in the electric field. Figures 2(a)-2(c) show the formation free energy surfaces $\left(\Delta G_{i+1, v} / k_{\mathrm{B}} T\right.$ as a function of $i$ and $v)$ at zero field, and at $E=0.4\left(\sigma^{3} / \epsilon\right)^{1 / 2}$ and $0.8\left(\sigma^{3} / \epsilon\right)^{1 / 2}$, respectively. The monomer pressure $p_{1}$ $=N_{1} k_{\mathrm{B}} T / V_{1}$ is chosen such that $p_{1} / p_{\text {eq }}$ is maintained at 2.5. It should be noted that the coexistence properties of the Stockmayer fluid change with the electric field. ${ }^{18,19}$ For example, the vapor density and pressure decreases with the electric field, and the critical temperature increases with the electric field. $p_{\text {eq }}$ of the Stockmayer fluid in the electric field are taken from Boda et al.'s simulation. ${ }^{19}$ Figures 3(a)-3(c) show $\Delta G_{i+1, v} / k_{\mathrm{B}} T$ as a function of $i$ at $v=200 \sigma^{3}, 500 \sigma^{3}$, and $800 \sigma^{3}$, respectively. We found that $\Delta G_{i+1, v} / k_{\mathrm{B}} T$ at the highest field $E=0.8\left(\sigma^{3} / \epsilon\right)^{1 / 2}$ exhibits the highest ridge

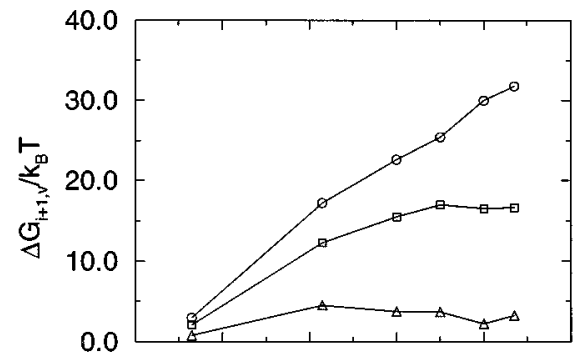

(a)

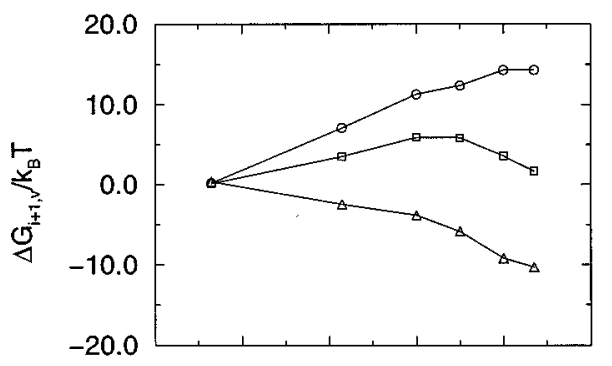

(b)

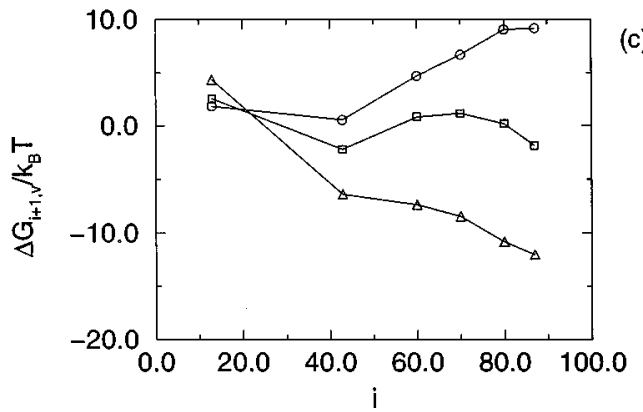

FIG. 3. $\Delta G_{i+1, v}$ as a function of $i$ for (a) $v=200 \sigma^{3}$, (b) $v=500 \sigma^{3}$, and (c) $v=800 \sigma^{3}$. Electric field strengths are $E=0.0\left(\sigma^{3} / \epsilon\right)^{1 / 2}$ (triangle up), $0.4\left(\sigma^{3} / \epsilon\right)^{1 / 2}$ (square), and $0.8\left(\sigma^{3} / \epsilon\right)^{1 / 2}$ (circle).

height, implying that under this field the critical cluster is the largest, compared to the other two cases. This result is apparently opposite to the previous ones ${ }^{5-7}$ predicted from the continuum theory. Recently, Warshavsky and Shchekin ${ }^{7}$ also found that at a fixed supersaturation a uniform electric field inhibits nucleation, which is the same as our prediction, although they used a different approach.

Let us consider a simple case: a spherical dielectric drop surrounded by a supersaturated vapor with dielectric constant $\epsilon_{g}$. From the continuum theory, the free energy change $\Omega_{E}$ of the drop due to the presence of the electric field $\mathbf{E}_{g}$ is given by ${ }^{20}$

$$
\Omega_{E}=-\frac{1}{8 \pi} \int \mathbf{E}_{g} \cdot\left(\mathbf{D}_{l}-\epsilon_{g} \mathbf{E}_{l}\right) d V,
$$

where $\mathbf{D}_{l}$ and $\mathbf{E}_{l}$ is the electric displacement and the electric field inside the drop, respectively. One may consider $\Omega_{E}$ as the formation free energy contribution due to the presence of the electric field. The formation free energy of a critical nucleus is then given by 
(a)

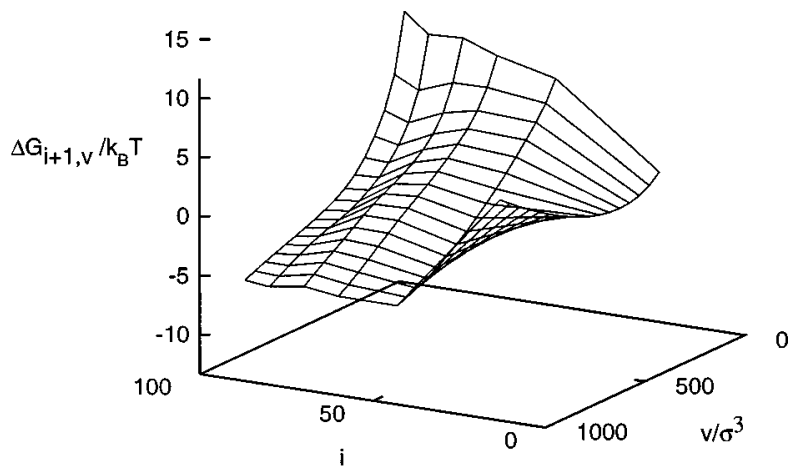

(b)

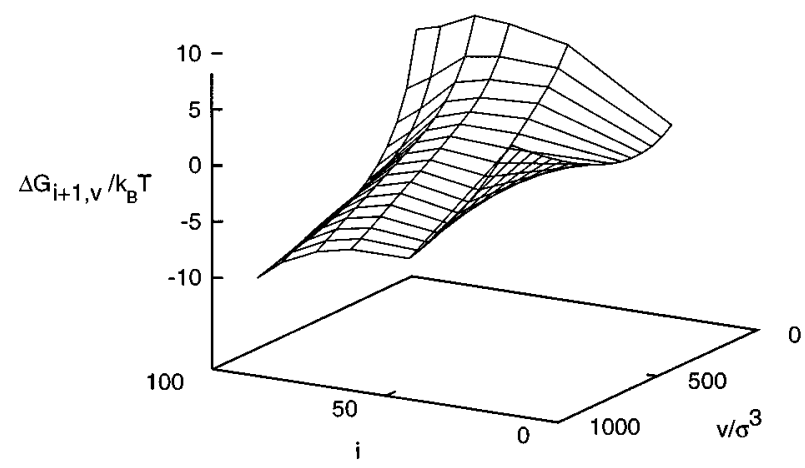

(c)

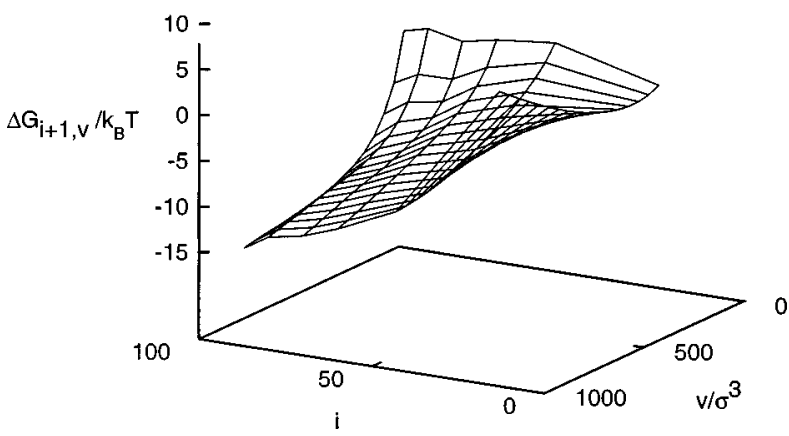

FIG. 4. The same as Fig. 2, but $\mu_{1}$ is set to $-18.23 \epsilon$.

$$
\Delta \Omega^{*}=\frac{16 \pi}{3} \frac{\sigma^{3}}{\Delta p+\alpha E_{g}^{2}},
$$

where $\sigma$ is surface tension, $\Delta p$ is the pressure difference between the drop and the vapor, and $\alpha=3 \epsilon_{g}\left(\epsilon_{l}\right.$ $\left.-\epsilon_{g}\right) / 8 \pi\left(2 \epsilon_{g}+\epsilon_{l}\right) . \alpha$ is normally positive because $\epsilon_{l}$ is larger than $\epsilon_{g}$. At the condition that $\Delta p$ is fixed as used in the previous studies, ${ }^{5-7}$ the formation free energy of the critical nucleus is decreased by the electric field. At the same condition (fixed $\Delta p$ ), the supersaturation $S$ is increased by the electric field because $\ln S=\left(\Delta p+\alpha E_{g}^{2}\right) / \rho_{l} k_{\mathrm{B}} T$, where $\rho_{l}$ is the density of the drop. It was also assumed in the previous studies that the electric field does not affect $\sigma, \epsilon_{g}$, and $\epsilon_{l}$. In contrast, the condition used in this study (fixed $S$ ) is different from that used in the previous studies ${ }^{5-7}$ Here, we do not need to assume the independence of $\sigma, \epsilon_{g}$, and $\epsilon_{l}$ on the electric field. We note also that the classical nucleation theory employs the surface tension of a flat surface. How-

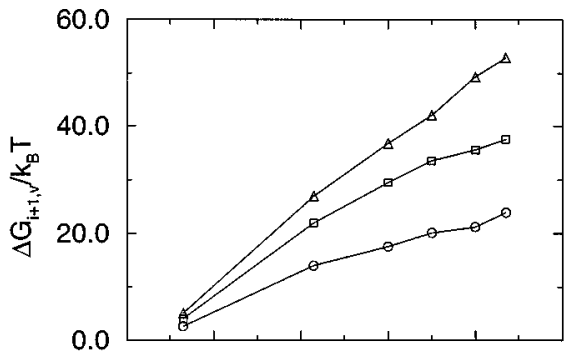

(a)

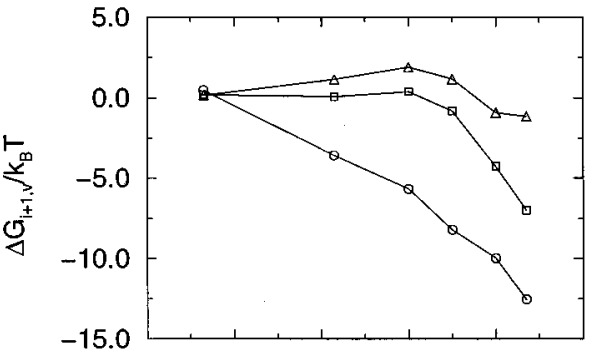

(b)

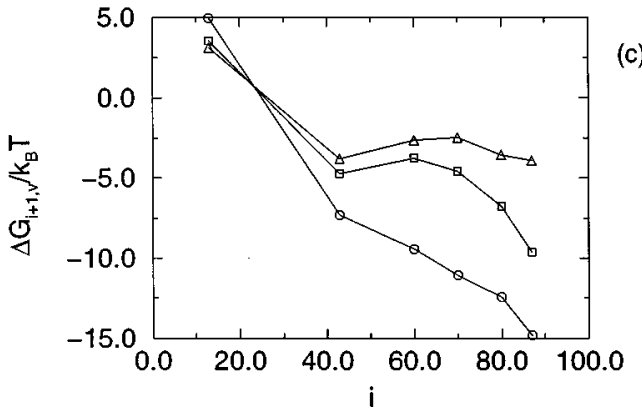

FIG. 5. The same as Fig. 3, but $\mu_{1}$ is set to $-18.23 \epsilon$.

ever, $\sigma$ of a flat surface in the electric field is different when a tangential or a normal field to the surface is applied. ${ }^{21}$ This difference may cause some problems when classical nucleation theory is used for vapor nucleation in the electric field.

Next, we consider the case of a fixed monomer chemical potential. This is usually accomplished in a grand canonical ensemble simulation, such as the grand canonical Monte Carlo simulation proposed by Kusaka et al. ${ }^{22,23}$ Figures 4(a)-4(c) show formation free energy surfaces at fixed chemical potential, $\mu_{1}=-18.23 \epsilon$, and at $E=0.0\left(\sigma^{3} / \epsilon\right)^{1 / 2}$, $0.4\left(\sigma^{3} / \epsilon\right)^{1 / 2}$, and $0.8\left(\sigma^{3} / \epsilon\right)^{1 / 2}$, respectively. To maintain the same chemical potential $\left(\mu_{1}\right)$, it is necessary for the monomer density $N_{1} / V_{1}$ to increase with the electric field. This means that the supersaturation is effectively enhanced by the electric field because the coexistence vapor density decreases with the electric field, as indicated above. As shown in Figs. 5(a) $-5(\mathrm{c}), \Delta G_{i+1, v}$ decreases with the electric field. This behavior is also observed in the grand canonical Monte Carlo simulation of water in the electric field. ${ }^{24}$

\section{CONCLUSION}

The formation free energy of Stockmayer clusters in a uniform electric field is determined from the $i / v$ cluster theory in conjunction with Monte Carlo simulations. It is found that the formation free energy increases with the elec- 
tric field at fixed supersaturation $\left(p_{1} / p_{\text {eq }}\right)$ and decreases at a fixed chemical potential. In obtaining our results, we avoid the assumption that the surface tension, dielectric constant of the vapor and the cluster are independent of the electric field, which may cause a large error in predicting the formation free energy of the critical nucleus. This is a main advantage of the molecular-based approach. In addition, the shape of the cluster is partially taken into account in calculating $A_{i, v}^{*}$. Although we use a spherical container, the core region in the cluster may have a nonspherical average shape because there still exist vapor molecules outside a core region.

Based on ideal gas approximation of the supersaturated vapor, we assumed the interactions between the cluster and the vapor can be ignored. This makes it possible to simulate an isolated cluster (like an LBA cluster). When the temperature is well below the critical temperature, equilibrium vapor density as well as the dielectric constant of the vapor is very close to that of a vacuum. Therefore, an ideal gas approximation is reasonable. However, an ideal gas approximation may not hold at higher vapor densities (e.g., near the critical point), because there can be substantial intermolecular interactions and vapor-cluster interactions. Moreover, the dielectric constant of the vapor may not be close to that of the vacuum. We note in passing that a method to incorporate the interactions between the cluster and the surrounding vapor has been recently developed by Reiss and co-workers. ${ }^{25,26}$

In this work, we used a simple Stockmayer potential to model dipolar molecules so that polarization mainly arises from the orientational arrangement of the molecules along the electric field. Under very high electric fields, the molecular polarizability would also be an important factor of polarization. Thus, polarizable potential models are needed in order to account for that factor. Studies of vapor-liquid nucleation in the electric field using polarizable molecular models are underway.

\section{ACKNOWLEDGMENTS}

We are grateful to Professor Howard Reiss, Professor Alexander Shchekin, and Dr. Kiyohara and Dr. Koga for useful discussions. X.C.Z. thanks the National Science Foundation for support of this work.

${ }^{1}$ C. H. Chalmers, Atmospheric Electricity (Pergamon, Oxford, 1967).

${ }^{2}$ H. Rabeony and P. Mirabel, J. Chem. Phys. 83, 219 (1986).

${ }^{3}$ J. L. Katz, J. A. Fisk, and V. M. Chakarov, J. Chem. Phys. 101, 2309 (1994).

${ }^{4}$ D. Kashchiev, Philos. Mag. 25, 459 (1972).

${ }^{5}$ J. O. Isard, Philos. Mag. 35, 817 (1977).

${ }^{6}$ K. J. Cheng, Phys. Lett. 106, 403 (1984).

${ }^{7}$ V. B. Warshavsky and A. K. Shchekin, preprint.

${ }^{8}$ A. Y. Zubarev and A. O. Ivanov, Phys. Rev. E 55, 7192 (1997).

${ }^{9}$ D. Wright, R. Caldwell, C. Moxely, and M. S. El-Shall, J. Chem. Phys. 98, 3356 (1993).

${ }^{10}$ D. Wright and M. S. El-Shall, J. Chem. Phys. 98, 3369 (1993).

${ }^{11}$ H. M. Ellerby, C. L. Weakliem, and H. Reiss, J. Chem. Phys. 95, 9209 (1991).

${ }^{12}$ H. M. Ellerby and H. Reiss, J. Chem. Phys. 97, 5766 (1992).

${ }^{13}$ C. L. Weakliem and H. Reiss, J. Chem. Phys. 99, 5374 (1993).

${ }^{14}$ C. L. Weakliem and H. Reiss, J. Chem. Phys. 101, 2398 (1994).

${ }^{15}$ K. J. Oh, X. C. Zeng, and H. Reiss, J. Chem. Phys. 107, 1242 (1997).

${ }^{16}$ J. K. Lee, J. A. Barker, and F. F. Abraham, J. Chem. Phys. 58, 3166 (1973).

${ }^{17}$ C. J. F. Bottcher, Theory of Electric Polarization (Elsevier, Amsterdam, 1973), Vol. I.

${ }^{18}$ M. J. Stevens and G. S. Grest, Phys. Rev. E 51, 5976 (1995).

${ }^{19}$ D. Boda, J. Winkelmann, J. Liszi, and I. Szalai, Mol. Phys. 87, 601 (1996)

${ }^{20}$ L. D. Landau, E. M. Lifshitz, and L. P. Pitaevskii, Electrodynamics of Continuous Media (Pergamon, Oxford, 1984).

${ }^{21}$ L. Liggieri, A. Sanfeld, and A. Steinchen, Physica A 206, 299 (1994).

${ }^{22}$ I. Kusaka, Z.-G. Wang, and J. H. Seinfeld, J. Chem. Phys. 108, 3416 (1998).

${ }^{23}$ I. Kusaka, Z.-G. Wang, and J. H. Seinfeld, J. Chem. Phys. 108, 6829 (1998).

${ }^{24}$ G. Gao, K. J. Oh, and X. C. Zeng, in preparation.

${ }^{25}$ P. Schaaf, B. Senger, and H. Reiss, J. Phys. Chem. B 101, 8740 (1997).

${ }^{26}$ B. Senger, P. Schaff, D. S. Corti, R. Bowlew, D. Pointu, J.-C. Voegel, and H. Reiss, preprint. 